\title{
Behaviour of Mechanically Switched Capacitors with Damping Network (MSCDN) during Energization
}

\author{
S. Weck ${ }^{1}$, I. Talavera ${ }^{1}$ and J. Hanson ${ }^{1}$ \\ ${ }^{1}$ Institute of Electrical Power Systems with Integration of Renewable Energies \\ Technical University Darmstadt \\ Landgraf-Georg-Straße 4- 64283 Darmstadt (Germany) \\ Phone number: +00496151 16-76861, email: sebastian.weck@e5.tu-darmstadt.de
}

\begin{abstract}
Reactive Power Compensation is becoming increasingly important, as more and more conventional generation is replaced by dispersed energy sources, which do not contribute to voltage stability in the transmission grid. The Mechanically Switched Capacitors with Damping Network (MSCDN) is a costefficient solution, combining reactive power compensation and harmonic mitigation. In this paper, the MSCDN is analysed with respect to its design parameters to determine the electrical stress on the components during energization. The analysis is done in Laplace and frequency domain, as well as through time-domain numeric simulation.
\end{abstract}

\section{Key words}

Reactive Power, Harmonics, Voltage Stability, Power Transmission, C-type filter, Passive Filter.

\section{Introduction}

With more and more renewable energy sources penetrating into the German grid, the German transmission grid experiences an increasing amount of power to be transmitted from the windy Northern Germany to load centres in the South. Additionally, many gas- and coal-fired power plants have become unprofitable due to massive generation based on renewable energy. To make matters worse, nuclear power plants are in the process of being shut down. These facts lead to an increasing lack of reactive power, causing a risk of voltage instability in some regions of Germany.

To compensate this lack of reactive power, a common solution is shunt compensation based on mechanically switched capacitors (MSC). MSCs tend to cause resonances with the grid and must be designed to withstand all harmonics in steady state [1]. A solution to these disadvantages is the Mechanically Switched Capacitors with Damping Network (MSCDN). This component combines a capacitor with a damped single tuned filter. Therefore, the MSCDN can fulfill both the tasks of reactive power compensation as well as harmonic mitigation, while also avoiding the low-damped resonances with the connected network [2]. Moreover, the fundamental power losses occurring in conventional single tuned filters are removed.

The MSCDN is also a very economical solution, because it combines multiple tasks in single component without the use of power electronics.

During the design phase of the MSCDN, both steady state voltage stress and losses and transients during switching of the MSCDN have to be considered. There have been many investigations with different scope conducted, using various methods for the design of the $\operatorname{MSCDN}$ ([3], [4], [5], [6], [2]).

In order to analyse the transient behaviour of the MSCDN, an analytical approach as proposed by Horton et al. [5] has been adopted. The obtained results have been verified by numerical simulations of the switching transients.

\section{Theoretical Analysis of the MSCDN}

The MSCDN is based on a C-type filter topology. It consists of the main capacitor $C_{1}$, a single tuned filter consisting of filter capacitor $C_{2}$ and filter reactor $L$, and a damping resistor $R_{\mathrm{D}}$. The single line diagram of the MSCDN is shown in figure 1.

The single tuned filter is tuned to fundamental frequency $f_{1}$, while the entire MSCDN is tuned to a selectable tuning frequency $f_{\mathrm{t}}$. Thus, the MSCDN acts as a capacitor at fundamental frequency, while having small impedance at the tuned frequency. This makes the C-type filter topology useful for reactive power compensation as well as harmonic mitigation.

\section{A. Component Dimensioning}

The components of an MSCDN are solely defined be the system fundamental angular frequency $\omega_{1}=2 \pi f_{1}$, the rated voltage $U_{\mathrm{r}}$, the rated reactive power $Q_{\mathrm{r}}$, the tuning frequency order $h_{\mathrm{t}}=f_{\mathrm{t}} / f_{1}$ and the quality factor $q$. 


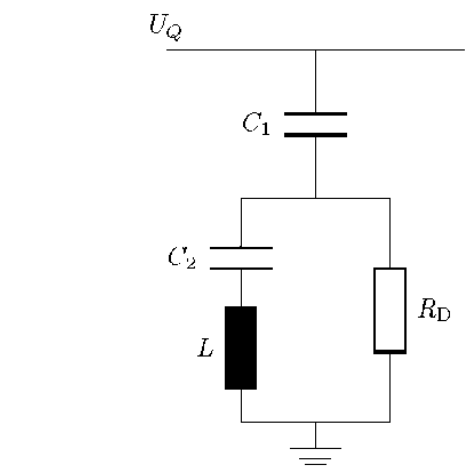

Figure 1: Single line diagram of an MSCDN

Using the design principle above, the values can be calculated by equations (1) - (4):

$$
\begin{aligned}
& C_{1}=\frac{Q_{\mathrm{r}}}{\omega_{1} U_{\mathrm{r}}^{2}} \\
& C_{2}=\frac{Q_{\mathrm{r}}\left(h_{\mathrm{t}}^{2}-1\right)}{\omega_{1} U_{\mathrm{r}}^{2}} \\
& L=\frac{U_{\mathrm{r}}^{2}}{\omega_{1} Q_{\mathrm{r}}\left(h_{\mathrm{t}}^{2}-1\right)} \\
& R_{\mathrm{D}}=\frac{q U_{\mathrm{r}}^{2}}{h_{\mathrm{t}} Q_{\mathrm{r}}}
\end{aligned}
$$

The quality factor can be interpreted as the damping resistance normalized to rated voltage, reactive power and tuning frequency, as described in equation (4). It is especially useful when comparing MSCDN with different design criteria.

\section{B. Frequency Response}

For the frequency domain analysis the components are viewed as complex, frequency-dependent impedances. By consideration of the complex impedance of grid and MSCDN, and subsequent application of a voltage divider, the complex transfer function of - for example - the voltage over the MSCDN $\underline{H}_{\mathrm{MSCDN}}(f)$ can be obtained as follows:

$$
\underline{H}_{\mathrm{MSCDN}}(f)=\frac{\underline{U}_{\mathrm{MSCDN}}}{\underline{U}_{\mathrm{Q}}}=\frac{\underline{Z}_{\mathrm{MSCDN}}(f)}{\underline{Z}_{\mathrm{Q}}(f)+\underline{Z}_{\mathrm{MSCDN}}(f)}
$$

with the parameters as shown in figure 2 .

This transfer function represents the frequency response of the MSCDN. By looking at the absolute value $|\underline{H}(f)|$ and the angle $\angle \underline{H}(f)$ of the frequency response, the behavior of the MSCDN during energization can be estimated. High absolute values of the transfer function at a given frequency lead to an amplification of that frequency during ener- gization. The maxima in the absolute value therefore represent the natural frequencies of the system. When the MSCDN is switched during maximum voltage, there will be an inrush current which excites the system. The system will then swing with its natural frequency. The angle of the transfer function determines the damping for each frequency.

The frequency response for each component of the MSCDN can be derived by using the basic equations for the impedances of the MSCDN's components. This approach has the advantage that frequency-dependent grid impedances can be easily taken into account. The frequency response of the individual components will be further described in chapter 3.

\section{Laplace-Domain Analysis}

The Laplace-domain transfer function $H(s)$ is obtainnd analogous to the complex function $H(s)=\underline{H}(\mathrm{j} \omega)$. analysing the poles of the transfer functions in Laplace domain, the dominant frequencies and their damping can be obtained. These poles can have a real form like in equation (6) or a complex conjugate form like in equation (7):

$$
\begin{gathered}
s=\alpha \\
s_{1,2}=-\rho \pm \mathrm{j} \mu
\end{gathered}
$$

Real poles represent DC quantities and complex poles represent resonating frequencies in the time response. The real part $\rho$ of the complex poles represents the damping, while the imaginary part $\mu$ determines the resonant frequency (in angular frequency). By an analysis of the poles for different quality factors, the influence of the damping resistor $R_{\mathrm{D}}$ can be analytically determined.

Using Laplace analysis, the behaviour of the MSCDN during energization can be determined in transformed as well as in time domain. The time-domain answer can be obtained by inverse Laplace transformation of the transfer function $H(s)$. This can be done by either using the Heaviside Theorem [7] or alternatively using numeric inverse Laplace transformation [8].

\section{Numerical Simulation of the Energization Process}

In addition to the symbolical analysis in frequency and Laplace domain, the system's differential equations can also be solved numerically. This paper uses the MATLAB SimPowerSystems toolbox for the numerical calculations. The advantages of a numerical solution are the simple layout of complicated models through a graphical user interface, predefined submodels for complex components and - most important - the possibility to easily specify the initial conditions for all components.

\section{E. Test Model and Study Cases}

The energization test model consists of a voltage source in series with the grid impedance, a breaker and the MSCDN. At time $t=t_{\text {in }}$, the breaker is switched on for varying values of the grid voltage. 


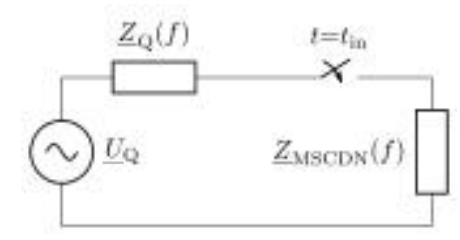

Figure 2: Test model equivalent circuit for the calculation of electrical stresses in an MSCDN

Table I. - Parameters of the Test Model

\begin{tabular}{cc}
\hline Parameter & Value \\
\hline$U_{\mathrm{Q}}$ & $420 \mathrm{kV}$ \\
$f_{1}$ & $50 \mathrm{~Hz}$ \\
$R_{\mathrm{Q}}$ & $0.5 \Omega$ \\
$X_{\mathrm{Q}}$ & $5 \Omega$ \\
$U_{\mathrm{r}}$ & $380 \mathrm{kV}$ \\
$Q_{\mathrm{r}}$ & $300 \mathrm{MVAr}$ \\
$f_{\mathrm{t}}$ & $200 \mathrm{~Hz}$ \\
$q$ & $0.01 \leq q \leq 10$ \\
\hline
\end{tabular}

For this study, a simplified positive sequence model has been chosen, as this is sufficient for the realized symmetric analysis. The equivalent circuit diagram of the test model is shown in figure 2.

For the analysis in this paper, the values specified in table I have been assumed. The behaviour during energization is evaluated for a range of the quality factor of $0.01 \leq q \leq$ 10. This is done to show the influence of the quality factor and therefore the damping resistance. For the chosen design parameters, these quality factors correspond to $R_{\mathrm{D}}=12.03 \Omega, 120.33 \Omega$ and $1203.33 \Omega$ respectively.

The behaviour of the MSCDN during energization is dependent on the frequency-dependent, complex impedances $\underline{Z}_{\mathrm{Q}}(f)$ for the grid and $\underline{Z}_{\mathrm{MSCDN}}(f)$ for the MSCDN itself. For different frequencies the grid impedance has different behaviour - capacitive, inductive or resistive - and significant resonances, which can and should be positively influenced through the application of MSCDN.

For transient analysis, the grid impedance is often given as a first order model that is also used for this work:

$$
\underline{Z}_{\mathrm{Q}}(f)=R_{\mathrm{Q}}+\mathrm{j} \omega_{1} L_{\mathrm{Q}}
$$

This assumption is appropriate for substations without significant resonances due to shunt capacitors or due to long overhead lines.

In this paper, three study cases have been carried out to evaluate the impact of the quality factor (table II):

1) Case 1: In this case, the results for frequency domain analysis methods mentioned in sections 2-B and 2-C are obtained and validated by numerical calculation. The switching is done during maximum voltage, because
Table II. - Summary of realized Study Cases

\begin{tabular}{cccc}
\hline & Case 1 & Case 2a & Case 2b \\
\cline { 2 - 4 } $\boldsymbol{U}_{\mathbf{Q}}\left(\boldsymbol{t}_{\text {in }}\right)$ & $\hat{U}_{\mathrm{Q}}$ & $\hat{U}_{\mathrm{Q}}$ & 0 \\
Pre-charge of $\boldsymbol{C}_{\mathbf{1}}$ & 0 & $-0.5 \hat{U}_{\mathrm{Q}}$ & $-0.5 \hat{U}_{\mathrm{Q}}$ \\
\hline
\end{tabular}

the system is strongly excited then. No pre-charging of the capacitors $C_{1}$ and $C_{2}$ is assumed.

For switching operations, the main capacitor $C_{1}$ of the MSCDN can still be partly charged. Therefore, for the following study cases, a pre-charging of the capacitor of $\mathbf{- 0 . 5} \hat{U}_{\mathbf{Q}}$ is assumed for two different switching instants:

1) Case 2a: The MSCDN is switched on at voltage maximum with a pre-charge of the main capacitor of $-0.5 \hat{U}_{\mathrm{Q}}$.

2) Case 2b: The MSCDN is switched on at zero voltage with a pre-charge of the main capacitor of $-0.5 \hat{U}_{\mathrm{Q}}$.

\section{Component Results}

\section{A. Study Case 1}

The study case 1 is performed to get an overview over the behaviour of the MSCDN during the energization process and to compare the analytical methods described in section 2. As the results for the frequency domain are similar, only the results for the voltage over the MSCDN are shown graphically in figure 3. Equivalent results can be obtained for the voltage and also for the currents at each component. The time responses of the voltages at the capacitors, the currents through the reactor and the power in the damping resistance are given in figure 4 .

1) Voltage over MSCDN: The impedance of the MSCDN in relation to the grid impedance determines the electrical stress of the station. The resulting total impedance $\underline{Z}_{\text {total }}=\underline{Z}_{\mathrm{Q}}+\underline{Z}_{\mathrm{MSCDN}}$ of the MSCDN and the grid is very important for the behavior of the MSCDN. Figure 3 shows the absolute value of the total impedance $\left|\underline{Z}_{\text {total }}(f)\right|$ for different quality factors. This figure shows the fundamental impact of the damping resistance on the behaviour of the MSCDN. When the quality factor is very low, the single tuned filter is effectively short circuited. When the quality factor is very high, the impedance value shows a very distinctive band-pass behaviour. The impedance thus has different minima at two frequencies, depending on the quality factor:

$$
\begin{gathered}
f_{\mathrm{r}, 1} \approx \frac{1}{\sqrt{L_{\text {total }} \cdot C_{\text {total }}}} \\
f_{\mathrm{r}, 2} \approx \frac{1}{\sqrt{L_{\mathrm{Q}} \cdot C_{1}}}
\end{gathered}
$$




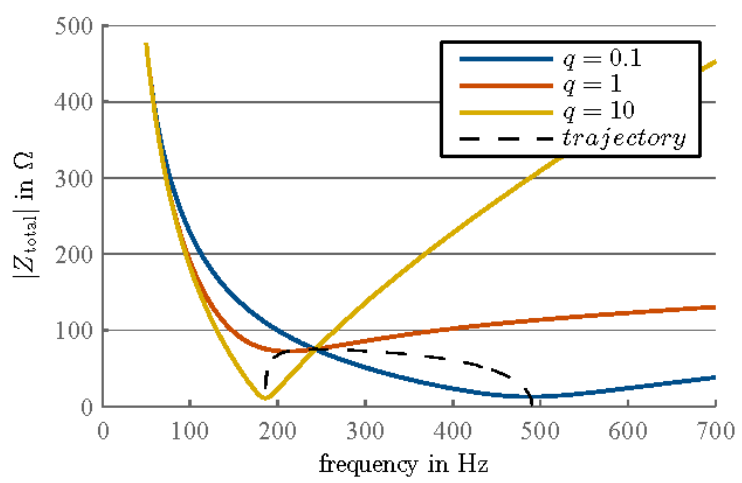

(a) Absolute value of $Z_{\text {total }}$ for different values of $q$

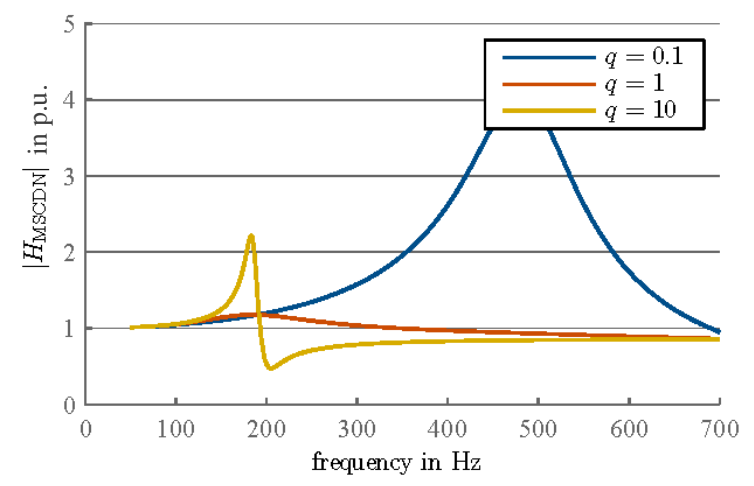

(c) Absolute value of $\underline{H}_{\mathrm{MSCDN}}(f)$ for different values of $q$

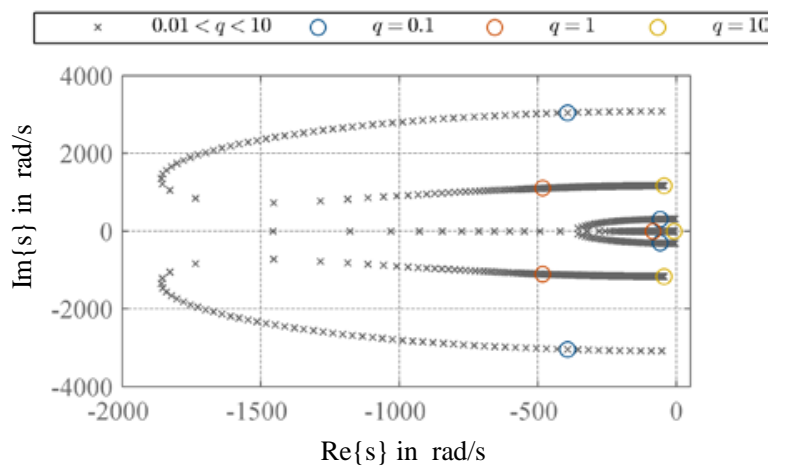

(b) Poles of the transfer function $H_{\mathrm{MSCDN}}$ for $0.01 \leq q \leq 10$

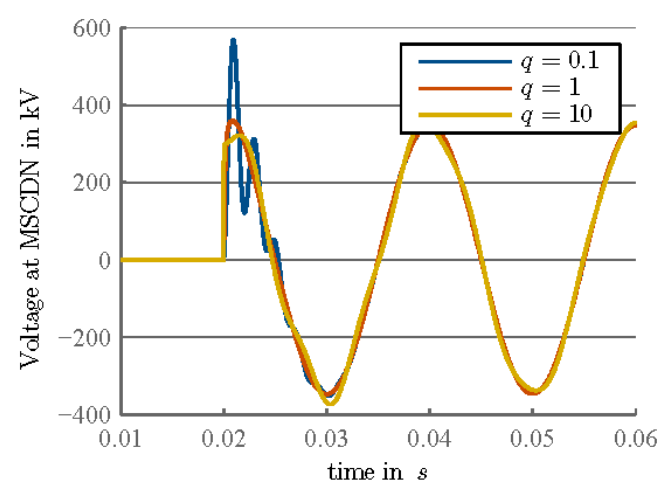

(d) Numerically-obtained time domain response for different quality factors

Figure 3: Study Case 1: Analysis of the voltage at MSCDN for the applied methods

$f_{\mathrm{r}, 1}$ is the resonant frequency when the quality factor is sufficiently high, and is located near the tuning frequency of the MSCDN.

When the quality factor is very low, the MSCDN is detuned, and the resonant frequency is only depending on the values of the main capacitor $C_{1}$ and the grid reactance (in this case $L_{\mathrm{Q}}$ ). For the design data used in this study, these frequencies are $f_{\mathrm{r}, 1}=186.6 \mathrm{~Hz}$ and $f_{\mathrm{r}, 2} \approx 480 \mathrm{~Hz}$. These frequencies are the respective minima of $\left|\underline{Z}_{\text {total }}(f)\right|$.

An analysis in Laplace domain yields the transfer function

$$
H_{\mathrm{MSCDN}}(s)=\frac{U_{\mathrm{MSCDN}}}{U_{\mathrm{Q}}}=\frac{Z_{\mathrm{MSCDN}}(s)}{Z_{\mathrm{total}}(s)}
$$

which has the poles shown in figure 3b. Analysis in frequency domain gives the transfer function $\underline{H}_{\operatorname{MSCDN}}(f)=$ $H_{\mathrm{MSCDN}}(s=j 2 \pi f)$ with the absolute value given in figure 3c. The results show, that for very low as well as for higher values of the quality factor, there is very low damping and a higher amplification at the resonant frequencies.

A very good damping as well as a resonant frequency near the nominal tuning frequency is obtained with a quality factor of around 1. Higher values yield lower damping, negatively influencing the transient response. Lower values yield even higher damping at first. However, the reso- nant frequency deviates from the tuning frequency quickly and the response becomes very sensitive to variances of the damping resistance. For very small values of $q$, the resonant frequency deviates against $f_{\mathrm{r}, 2}$ (as shown in equation (10)), detuning the MSCDN.

Figure 3d shows the results for the time-domain analysis numerical solution. The analytical results obtained by inverse Laplace transformation are the same as the numerical results. The two different resonant frequencies can be seen, resulting in higher peak values in time domain for very high and low quality factors.

2) Main Capacitor $C_{1}$ : The main capacitor $C_{1}$ is the main component of the MSCDN. It has to be dimensioned for a large part of the nominal voltage of the network and has to carry all of the operating current. It is therefore the most expensive component, and any additional stress makes up a relatively large increase in investment cost. The voltage stress at the main capacitor can be determined by its transfer function

$$
\underline{H}_{\mathrm{C}_{1}}(f)=\frac{\underline{U}_{\mathrm{C}_{1}}}{\underline{U}_{\mathrm{Q}}}=\frac{\underline{Z}_{\mathrm{C}_{1}}(f)}{\underline{Z}_{\text {total }}(s)}
$$

This transfer function shows a qualitatively similar behaviour as $\underline{H}_{\mathrm{MSCDN}}$, with poorly damped poles and high amplification at $f_{\mathrm{r}, 1}$ and $f_{\mathrm{r}, 2}$ for high and very low quality factors respectively. 


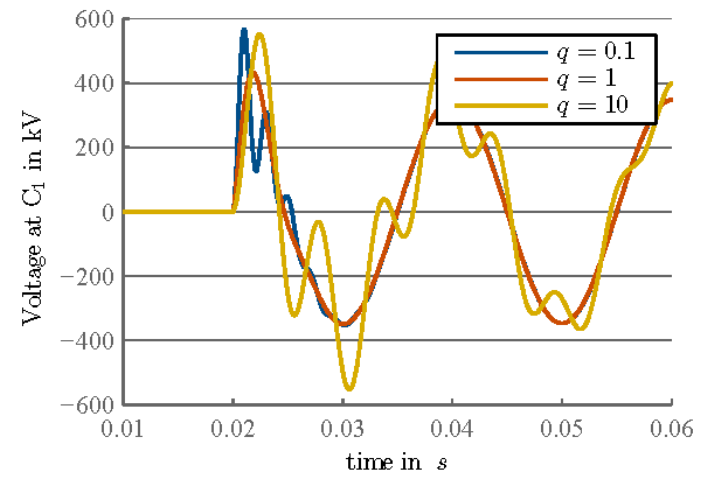

(a) Voltage at $C_{1}$

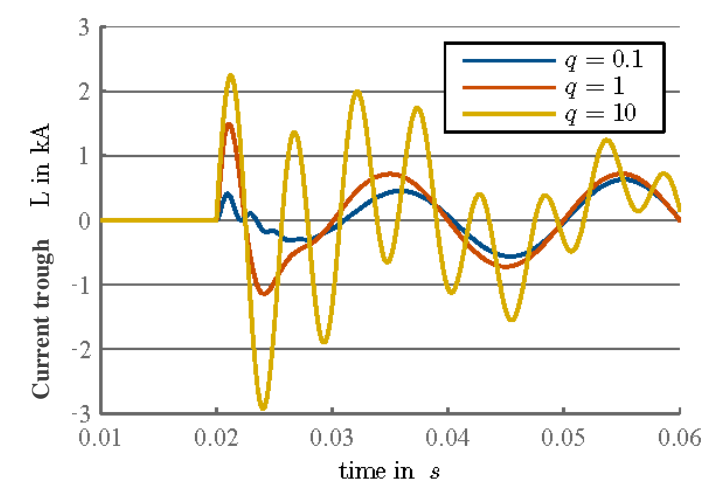

(c) Current through $L$

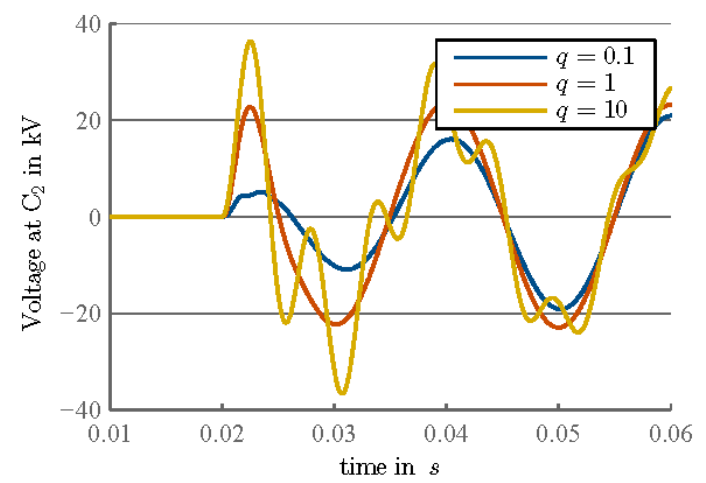

(b) Voltage at $C_{2}$

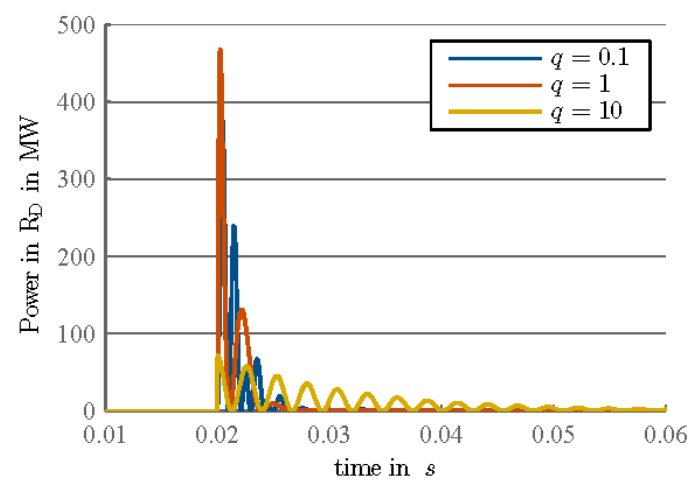

(d) Power in $R_{\mathrm{D}}$

Figure 4: Study Case 1: Time-domain responses for each component depending on $q$

The results of the time domain analysis for the energization are given in figure 4a. Remarkably, the influence of high quality factors on $U_{\mathrm{C}_{1}}$ is higher than for $U_{\mathrm{MSCDN}}$. As the main capacitor is the most crucial component, the big influence of high quality factors has to be considered especially.

3) Single tuned filter $\mathrm{L}, \mathrm{C}_{2}$ : The voltages and currents of the single tuned filter components $C_{2}$ and $L$ can be determined by using voltage and current dividers. The resulting transfer function for the voltage $U_{\mathrm{C}_{1}}$ and the current $I_{\mathrm{L}}$ are:

$$
\begin{gathered}
\underline{H}_{\mathrm{C}_{2}, U}(f)=\frac{\underline{U}_{\mathrm{C}_{2}}}{\underline{U}_{\mathrm{Q}}}=\frac{\underline{Z}_{\mathrm{C}_{2}}(f) \cdot \underline{Z}_{\text {filter }}(f)}{\underline{Z}_{\mathrm{STF}}(f) \cdot \underline{Z}_{\text {total }}(f)} \\
\underline{H}_{\mathrm{L}, I}(f)=\frac{\underline{I}_{\mathrm{L}}}{\underline{U}_{\mathrm{Q}}}=\frac{\underline{Z}_{\mathrm{filter}}(f)}{\underline{Z}_{\mathrm{STF}}(f) \cdot \underline{Z}_{\text {total }}(f)}
\end{gathered}
$$

$\underline{Z}_{\text {STF }}$ is the frequency-dependent impedance of the single tuned filter, $\underline{Z}_{\text {filter }}$ of the damped filter. These transfer functions also have similar poles as (11) and (12). Additionally, as the filter is tuned to $f_{1}$, there are poles on the imaginary axes for the fundamental frequency. The resulting time-domain responses are given in figure $4 \mathrm{~b}$ and figure 4c. The influence of extreme quality factors on switching voltage of $C_{2}$ is high as well. The voltage and current behaves differently, in that very low quality factors lead to low energization voltages and currents, because the single tuned filter is effectively short circuited.

4) Damping resistance $R$ : The damping resistance is used to achieve a desired quality factor for the MSCDN. In steady state, there is no fundamental current over the resistor, as the impedance of the single tuned filter is zero for the fundamental frequency. During energization there is a transient current in the resistor. In this case, it is beneficial to investigate the power in the damping resistor, because the design criterion for the resistor is mainly the energy dissipated in the resistor.

Figure 4d shows the power in the damping resistor during energization. The value of the damping resistor is proportional to the quality factor. Therefore lower quality factors cause high currents in $R_{\mathrm{D}}$. For high quality factors there are very low currents with smaller damping. Because the power is proportional to the square of the current and proportional to the resistor value, the power in the resistor during energization shows high peaks with high damping for low quality factors and vice versa. Because the energy dissipated is based on the integration of the power in the damping resistor, the losses are comparable for all quality factors. The losses in $R_{\mathrm{D}}$ are shown in figure 5 . The energy varies by approximately $6 \%$ over the range of $q$. Only a small increase for low quality factors is observed. 


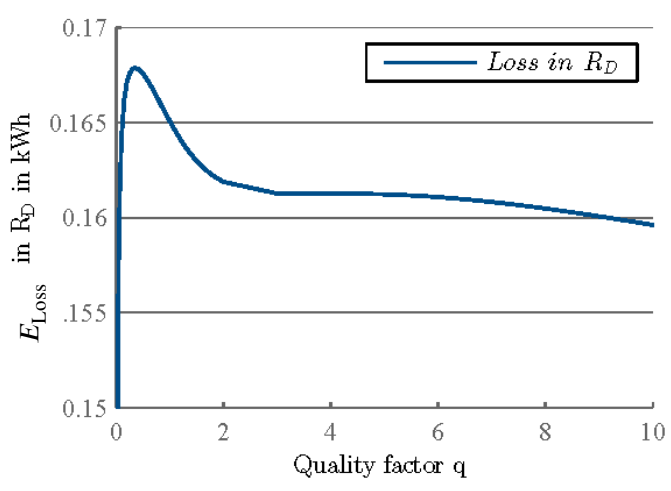

Figure 6: Case 1: Energy $E_{\text {Loss }}$ dissipated in the damping resistance $R_{\mathrm{D}}$ depending on the quality

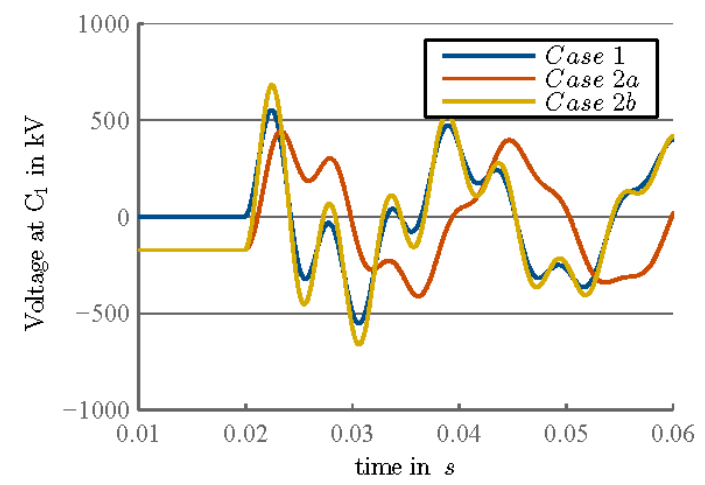

Figure 6: Comparison of study cases for $q=10$

5) Summary: The results show that to achieve a minimum peak value during energization while still preserving the design criteria of the MSCDN, a quality factor around 1 should be chosen.

\section{B. Study Cases $2 a$ and $2 b$}

The study cases $2 \mathrm{a}$ and $2 \mathrm{~b}$ are used to represent switching operations, where - as a worst case scenario - the capacitor $C_{1}$ is partly charged. The results for these cases show, that the results are comparable to study case 1 . A comparison of the switching voltages for $q=10$ over the main capacitor $C_{1}$ for all study cases is shown in figure 6 . The resonances and damping at all components are the same for all cases, with lower voltages for case $2 \mathrm{a}$ and higher voltages for case $2 b$.

For case 2a, the switching is done during zero voltage, therefore the voltage behaves differently than in the other cases. The resulting peaks are lower than for case 1 , because the pre-charging of the capacitor is not as influential as the phase of the grid voltage at switching.

The result for case 2b shows the same behavior of the voltage, but yields higher peaks than in case 1 . This is because of the additional pre-charge, which leads to a higher initial voltage over $C_{1}$.

\section{Conclusion}

In this paper, the MSCDN's behaviour during energization is analysed using Laplace-, frequency- and timedomain analysis. The results are evaluated for a wide range of quality factors and three different study cases. The lowest peaks and best damping are achieved by using quality factors around 1 for all cases. The transient voltages occurring over the MSCDN and its components are getting higher and less damped for very high and low quality factors. This has to be considered when designing the MSCDN. For a complete analysis during the design phase, the transient and steady-state stresses as well as the desired harmonic mitigation and the grid resonances have to be taken into account and balanced against each other.

\section{Outlook}

The results obtained in this paper suggest an ideal quality factor $q$ around 1 . This should be verified for different design criteria, e.g. tuning frequency or rated reactive power. Moreover, the influence of realistic grid impedances with resonances on the switching transient should be examined as described in [9]. Finally, the influence of non-ideal components due to losses and tolerances on the obtained results in steady and transient state should be analysed.

\section{References}

[1] J. Enslin, J. Knijp, C. Jansen, and J. Schuld, "Impact of reactive power compensation equipment on the harmonic impedance of high voltage networks," in Power Tech Conference Proceedings, 2003 IEEE Bologna, vol. 2, 2003.

[2] R. C. Campos, D. O. Lacerda, and M. Alves, "Mechanically switched capacitor with damping network (mscdn) engineering aspects of application, design and protection," in Transmission and Distribution Conference and Exposition: Latin America (T D-LA), 2010 IEEE/PES, Nov 2010, pp. 316-322.

[3] C.-J. Chou, C.-W. Liu, J.-Y. Lee, and K.-D. Lee, “Optimal planning of large passive-harmonic-filters set at high voltage level," vol. 15, no. 1, 2000, pp. 433-441.

[4] H. Zimmer, J. Dragon, and J. Hanson, "Basic design considerations for a mechanically switched capacitor with damping network (mscdn) within a high-voltagegrid," in Power Electronics and Applications (EPE), 2013 15th European Conference on, Sept 2013, pp. 1-10.

[5] R. Horton, R. Dugan, and D. Hallmark, "Novel design methodology for c-type harmonic filter banks applied in hv and ehv networks," in Transmission and Distribution Conference and Exposition (T\&D), 2012 IEEE PES, 2012, pp. 1-6.

[6] H. Hamid, N. Harid, and A. Haddad, "Transient analysis during switching of mechanically switched capacitor and damping network (mscdn)," in Universities Power Engineering Conference (UPEC), 2010 45th International, Aug 2010, pp. 1-4.

[7] E. Slamecka and W. Waterschek, Schaltvorgänge in Hoch- und Niederspannungsnetzen. Siemens, 1972.

[8] J. Valsa and L. Brancik, "Approximate formulae for numerical inversion of laplace transforms," Int. Journal of Numerical Modelling: Electronic Networks, Devices and Fields, 1998.

[9] B. Gustavsen and A. Semlyen, "Rational approximation of frequency domain responses by vector fitting," vol. 14, no. 3, 1999, pp. 1052-1061. 